\title{
Joint Equalization and Decoding Scheme Using Modified Spinal Codes for Underwater Communications
}

\author{
Yupeng TAI \\ State Key Laboratory of Acoustics \\ Institute of Acoustics \\ Chinese Academy of Sciences \\ Beijing, China
}

\author{
Frédéric GUILLOUD, Christophe LAOT \\ and Raphaël LE BIDAN \\ SC department \\ Telecom Bretagne Institut Mines-Telecom \\ Brest, France
}

\author{
Haibin WANG \\ State Key Laboratory of Acoustics \\ Institute of Acoustics \\ Chinese Academy of Sciences \\ Beijing, China
}

\begin{abstract}
Intersymbol interference (ISI) cancellation over frequency-selective underwater acoustic channel (UAC) is usually performed efficiently using iterative equalization. To this aim, an interleaver is required between the error correction scheme and the modulation scheme, and the longer the interleaver block length the better the performance. However, in the case of timevarying UAC, the length of the interleaver should not be longer than the channel coherence time, thus limiting the performance. Hence, for time-varying UAC with a low coherence time, small interleaver block length have to be used. In this paper, we propose a small block length coding scheme and its associate joint equalization and decoding algorithm based on Spinal codes. We show its efficiency over static large delay spread channels and static UAC: more precisely, simulation results show that the proposed scheme achieves a higher bit error rate than the Turbo equalization scheme with the same block length, especially for high spectral efficiency modulations. Simulations of proposed scheme over UAC obtained from underwater sea experiments are also presented which show the promising performances.
\end{abstract}

\section{INTRODUCTION}

Underwater Acoustic Channels (UACs) are known as to be very difficult telecommunication channels, due to timevarying long intersymbol interferences (ISI), making ISI cancellation an active topic for UAC digital communications. Turbo equalization [1] can be performed to improve the error rate performance for ISI-transmissions: an interleaver is implemented between the error correction scheme and the modulation. In a turbo-equalization receiver, the performance benefits from the interleaver gain through an iterative process between the equalizer and the decoder. The equalizer produces the estimates of the transmitted symbols using different methods. The maximum likelihood (ML) estimation is the optimal method in getting the minimum bit error rate (BER). However, the complexity of such methods is often too high to be implemented, especially for channels with large delay spread like UACs. Lower complexity equalizer like linear optimization algorithms are then implemented, such as zero forcing (ZF) or minimum mean squared error (MMSE) estimation. Results shows that the linear MMSE (LE-MMSE) turbo equalizer could completely remove the ISI for a time-invariant frequency-selective channel with an acceptable complexity [2],
[3], providing that the interleaver block length is sufficiently long as the delay spread of the considered UAC is high. On the other hand, for time-varying channels, increasing the interleaver block length above the coherence time of the channel will occur a dramatic loss in performance since the channel is usually assumed to be constant for the block length for non-adaptive equalization which is considered in this paper. So the question is: how to improve the equalization performance when the coherence time of the UAC is low, if the interleaver length cannot be increased?

In this paper, a novel joint non-adaptive equalization and decoding scheme based on a modified fixed-rate spinal code is proposed. It aims to provide an acceptable performance for a large range of channels using short block-length codes. Spinal codes have been proposed recently in [4] as a new class of non-linear error correction codes based on random number generators and pseudo-random hash functions. There are two aspects within spinal codes which are quite different from popular channel codes. First, an invertible hash function is used which makes graphical or algebraic decoding impossible: the decoding method of spinal code is based on a tree structure algorithm. As the powerful mixing effect of hash function, the near-ML decoder is quite efficient. This is also the foundation of the proposed near-ML joint equalization and decoding method achieve an interesting performance-complexity tradeoff even for long delay spread channels. The second aspect is that the decoding complexity is not increasing when the constellation size of the modulation is increasing. These two features of spinal codes make them a good candidate for joint decoding and equalizing in a single pass, that is to say without the need of iterating the decoding process.

The remainder of this paper is organized as follows: in Section II, the transmission scheme and the notations are introduced, together with the encoding of Spinal codes. In Section III, the joint decoder and equalizer is presented, and the performance results are detailed in Section IV. Some conclusions and perspectives are drawn in Section V. 


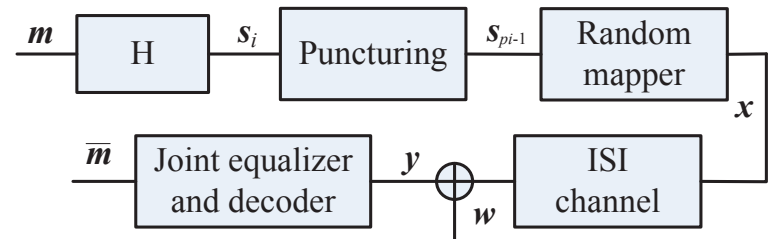

Fig. 1. The transmission scheme

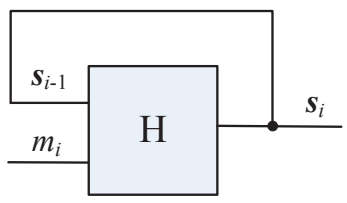

Fig. 2. The core hash structure

\section{The Transmission Scheme}

The transmission scheme is illustrated in Fig.1. Though spinal codes were proposed as rateless codes, we restrict the spinal code encoder to a fixed code rate denoted $R$. Length- $k$ blocks of independent information bits $\boldsymbol{m}$ are fed to a hash function denoted $\mathrm{H}$ which produces $k$ length- $v$ binary vector $s_{i}, 1 \leq i \leq k$, referred to as the seeds. The generation of the seeds can be implemented as an iterative process illustrated in Fig.2. The $i$-th bit of a message, $m_{i}$ is fed to the $\mathrm{H}$ function which is seeded by $s_{i-1}$. The output is seed $s_{i}$. The process is initiated by seed $s_{0}$ which is common to all messages $\boldsymbol{m}$ to be encoded and which is known by the receiver. The set of seeds $s_{i}$ is then punctured by a factor $p$, meaning that one seed every $p$ seeds is forwarded to the next transmitter function. Hence the transmitted seeds are $s_{p i-1}$ for $1 \leq i \leq k$. After puncturing, the remaining seeds are used to feed a random number generator (RNG) to generate $q$ symbols per seed, denoted $\boldsymbol{x}_{i}=\left\{x_{i, j}\right\}, 1 \leq j \leq q$ for seed $\boldsymbol{s}_{i}$. We assume that the cardinal of the constellation is denoted by $2^{c}$, i.e. each symbol $x_{i, j}$ represents $c$ bits. One possible solution to generate the symbols to be transmitted is illustrated in Fig.3 where the lowest significant bits of the random numbers are mapped to an I/Q constellation mapper.

The coding rate of the transmission is given by $R=$ $k p / c q k=p / c q$, and can thus be tuned over a wide range by varying the puncturing rate $p$, the constellation size $2^{c}$ or the number of symbols per seed $q$. Fig.4 illustrates the case where $k=6, p=3$ and $q=3$, that is a code rate $R=1 / c$.

The symbols $x_{i, j}$ are transmitted over a frequency-selective channel and corrupted by additive white Gaussian noise (AWGN) samples $w_{n}$ with variance $\sigma_{w}^{2}$. Let $\boldsymbol{x}$ be the concatenation of all the symbol vectors $\boldsymbol{x}_{i}$, and let $\boldsymbol{y}$ be vector of all the corresponding received samples. The received signal reads:

$$
y_{n}=\sum_{l=0}^{L-1} h_{l} x_{n-l}+w_{n}
$$

where $h_{l}$ are the coefficients of the discrete channel impulse response of length $L, x_{n}$ and $y_{n}$ are the $n$-th element of vector $\boldsymbol{x}$ and $\boldsymbol{y}$ respectively, corresponding to the transmission of message $\boldsymbol{m}$.

At the receiver side, the signal-to-noise ration is defined as:

$$
\mathrm{SNR}=\frac{E_{s}}{N_{0}}
$$

where $E_{s}$ is the average energy of $y_{n}-w_{n}$ and where $N_{0}$ is the mono-lateral power spectral density of the Gaussian noise.

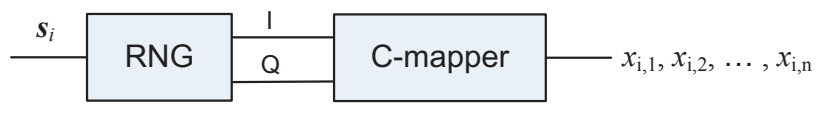

Fig. 3. The random mapper

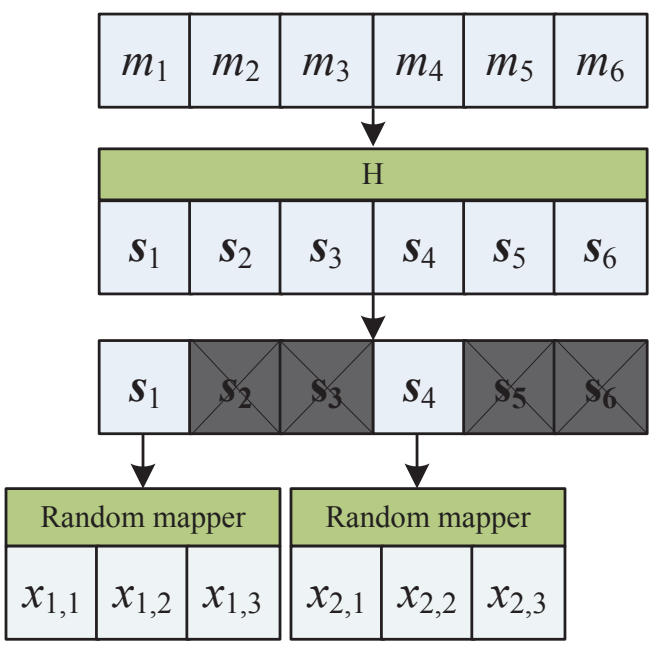

Fig. 4. The puncturing structure

\section{Joint Decoding AND Equalization Scheme}

\section{A. Near-ML Spinal Code Decoder}

The best error rate can be obtained by maximum likelihood (ML) decoding when the transmitted information is uniformly distributed. ML decoding for sequences corrupted by AWGN is obtained by minimizing the Euclidean distance between the received sequence and all the possibly transmitted sequences, that is:

$$
\widehat{\boldsymbol{m}}=\underset{\boldsymbol{m} \in\{0,1\}^{k}}{\arg \min }\|\boldsymbol{y}-\boldsymbol{x}(\boldsymbol{m})\|^{2}
$$

However the number of operations of ML decoding scales exponentially in $\mathcal{O}\left(2^{k}\right)$ with the information block length. Thus sub-optimal decoding algorithms are of interest. A suboptimal algorithm for spinal codes decoding is also proposed in [4] which is inspired by a popular complexity reduction named M-algorithm [5]. The idea is to replay the spinal code encoding process as a bit-wise tree structure. For each new binary information to be encoded, two branches are defined (corresponding to the 0 or 1 possible value) connecting the same tree parent-node to 2 child leaves. For each child-leaf in 


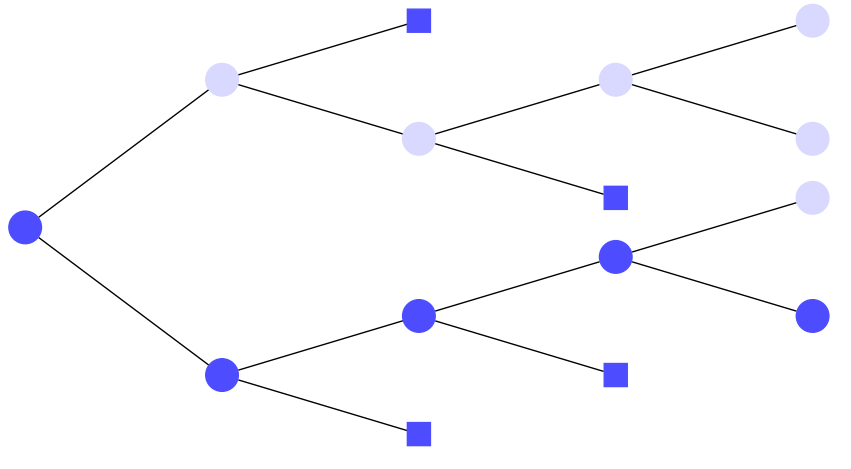

Fig. 5. Tree structure of decoder

$i$-th level of the tree, a partial Euclidean distance $D_{i}$ can be computed following the Equ.(4).

$$
D_{i}=\left\|\mathbf{y}_{i}-\mathbf{x}_{i}\left(s_{i}^{\prime}\right)\right\|^{2}+D_{i-1}
$$

where $\mathbf{y}_{i}$ and $\mathbf{x}_{i}\left(s_{i}^{\prime}\right), 1 \leq i \leq k$ are the sub-vectors of $\boldsymbol{y}$ and the candidate encoded vector containing symbols from the candidate seed $s_{i}^{\prime}$. For each new information bit, the number of leaves is multiplied by a factor 2 , resulting in $2^{k}$ leaves in the final tree.

Since the minimum Euclidean distance is to be found, a complexity reduction can be offered by selecting the leaves corresponding to the $B$ lowest Euclidean distances at each new information bit replayed. Fig.5 illustrates the complexity reduction algorithm when $B=2$ and $k=4$ : the light blue circular nodes represent the nodes corresponding to the lowest partial Euclidean distances, the dark blue square ones represent the pruned leaves (when the partial Euclidean distance is too high). The result of the decoder is then the sequence of bits corresponding to the minimum Euclidean distance among the $B$ remaining leaves of the last information bit, and it is illustrated by the dark blue circular nodes in Fig.5.

The $M$-algorithm is simple but very efficient in decoding Spinal codes. This is mainly due to the non-linearity and the use of random generator and hash functions which make it possible to increase the distance between two coded sequences corresponding to two messages where most of the bits are identical.

\section{B. The Near-ML joint Decoding and Equalization}

The tree-based low complexity decoding algorithm described in Section III-A can be easily adapted to include the equalization step thanks to the knowledge of the channel coefficients which are assumed to be estimated perfectly. Based on Eq.(4) and without considering any seed puncturing $(p=1)$, further break the sub-vectors $\mathbf{y}_{i}$ into symbols $y_{j}, q(i-1)+1 \leq j \leq q i$ and so as the $\mathbf{x}_{\left(s_{i}^{\prime}\right)}$, the partial Euclidean distance of the $i$-th level leaves of the tree change into:

$$
D_{i}=\sum_{j=q(i-1)+1}^{q i}\left|y_{j}-\sum_{l=0}^{L-1} h_{l} x_{j-l}\left(s_{t}^{\prime}\right)\right|^{2}+D_{i-1}
$$

TABLE I

NUMBER OF REQUIRED OPERATIONS PER SYMBOL OF PROPOSED SCHEME

\begin{tabular}{|c||c|}
\hline operation & amount \\
\hline hash\&RNG & $2 B(p+q) / q$ \\
\hline multiplications & $2 B L$ \\
\hline comparison & $\mathcal{O}(2 B p / q)$ \\
\hline
\end{tabular}

where $t=\left\lfloor\frac{j-l}{q}\right\rfloor+1$.

This joint decoding algorithm requires $k$ steps of expanding the tree. Each step expands $2 B$ leaves including one hash computations and one Euclidean distance computation. $k / p$ steps involve $q$ random number generations, $L q$ multiplications to estimate the channel convolution. The selection for the best $B$ candidates of each step requires $\mathcal{O}(2 B)$ operations. The number of required operations per symbol is given in table I.

Interestingly, if $B$ is kept constant, the complexity is linear in the message block length $k$ and independent of the number of bit per symbol $c$. Hence, high-order modulations can be implemented without increasing the number of Euclidean distance to compute.

In comparison, Turbo equalization requires several iterations between the BCJR [6] decoder and LE-MMSE equalization or MAP equalization. The BCJR algorithm for convolution decoder requires $\mathcal{O}\left(2^{\bar{M}} k\right)$ operations where $\bar{M}$ is the memory length of the code. LE-MMSE equalization requires $\mathcal{O}\left(\left(N^{2}+L^{2}\right) k\right)$ operations, where $N$ is the filter length. The MAP equalization required $\mathcal{O}\left(2^{c L} k\right)$ operations. With a proper selection of parameters, the proposed scheme requires a number of operations per symbols comparable to the LSMMSE Turbo equalization and much more efficient than the MAP Turbo equalization especially when the channel delay is large.

\section{Simulation Results}

\section{A. Universal Frequency-selective Channel}

The universal frequency-selective channels used in this section are proposed in [7]:

$$
\begin{aligned}
H_{\text {Proakis A }}= & {[0.04,-0.05,0.07,-0.21,-0.5,} \\
& 0.72,0.36,0,0.21,0.03,0.07]^{T} \\
H_{\text {Proakis B }}= & {[0.417,0.815,0.407]^{T} } \\
H_{\text {Proakis C }}= & {[0.227,0.460,0.688,0.460,0.227]^{T} }
\end{aligned}
$$

whose amplitude-frequency response is showed in Fig. 6.

The parameters for the proposed joint decoding and equalization using spinal codes are $k=96, B=1024, q=2, p=2$, yielding a code rate $R=1 / 2$. Comparison with turboequalization is provided to assess to performance-complexity trade-off of our proposed scheme. To this aim, a rate $-1 / 2$ 163,135 (expressed in octals) convolution code whose memory length is 6 has been implemented. QPSK signaling is used for both schemes.

Fig. 7, Fig. 8 and Fig. 9 present the performance of the proposed scheme and comparison with Turbo equalization 

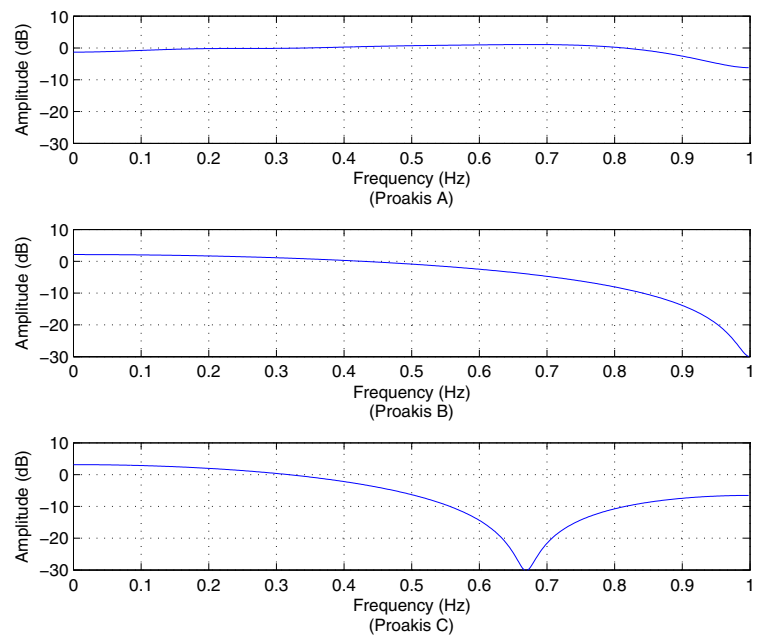

Fig. 6. The amplitude-frequency responses of the Proakis channel A,B and $\mathrm{C}$

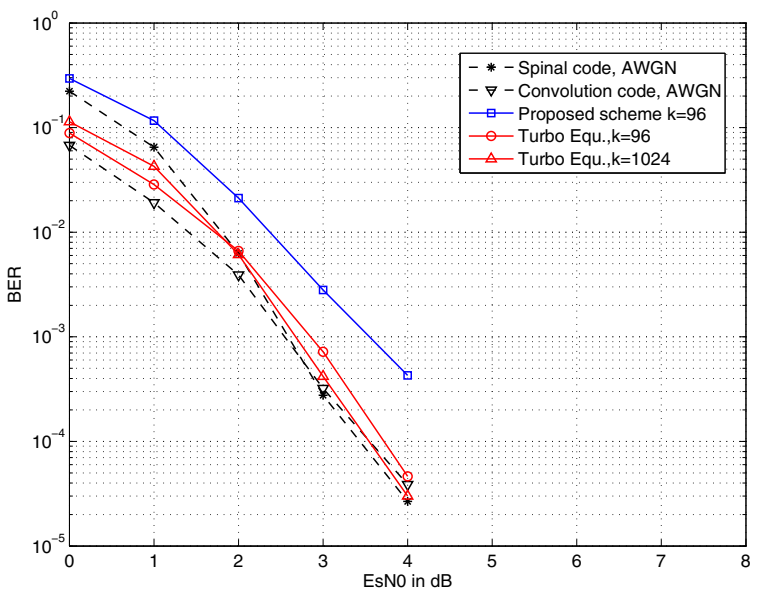

Fig. 7. The performance of proposed scheme and Turbo equalization over Proakis A channel for QPSK modulation

after 5 iterations over Proakis A,B and $\mathrm{C}$ channel respectively. The results show that the proposed scheme outperforms turbo-equalization when the block length is short for highly frequency-selective channels. However, a performance loss to the theoretical bound is observed with the lower frequencyselective channel, due to the sub-optimal equalization.

Fig. 10 shows the performances of the proposed scheme for 16-QAM signaling and its comparison with Turbo equalization. As $c$ is increased, the number of symbols per seed is set to $q=1$ to keep the code rate $R=1 / 2$. We can see that the performance gap between our proposed scheme and Turbo equalization over Proakis B and Proakis C channel increases compared to QPSK signaling. For these highly frequencyselective channels, it becomes more difficult for the Turbo equalization to get a reliable feedback of the estimated data

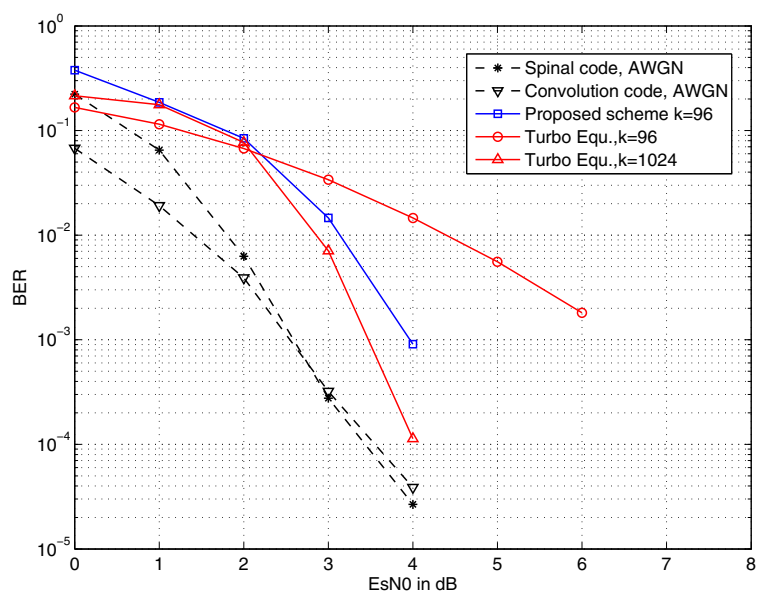

Fig. 8. The performance of proposed scheme and Turbo equalization over Proakis B channel for QPSK modulation

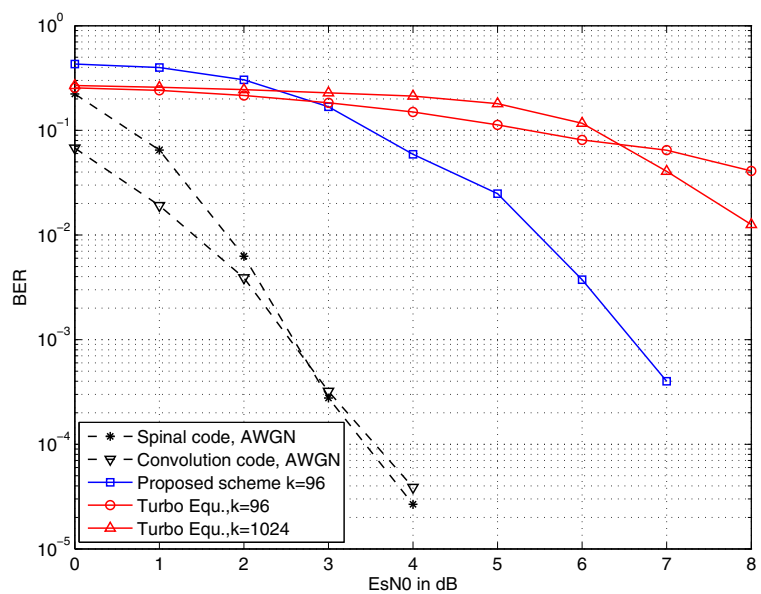

Fig. 9. The performance of proposed scheme and Turbo equalization over Proakis C channel for QPSK modulation

for low SNR, which is not the case for the porposed scheme.

\section{B. Underwater Acoustic Channels Extracted from Sea Exper- iment}

1) Shallow Sea Underwater Acoustic Channel: This channel is estimated from the data of an underwater acoustic communication experiment carried out on the South China Sea in 2011 by the Institute of Acoustic (IOA), Chinese Academy of Sciences (CAS). The channel coefficients and amplitude-frequency responses are represent in Fig. 11. The channel amplitude-frequency response is smooth except for a notch at the normalized frequency 0.2 . The performance of the proposed scheme and the Turbo equalization over this channel is compared in Fig.12. The proposed scheme also shows stable performance with small block length.

2) Deep Sea Underwater Acoustic Channel: Fig.13 shows the deep sea channel extracted from the data of an experiment 


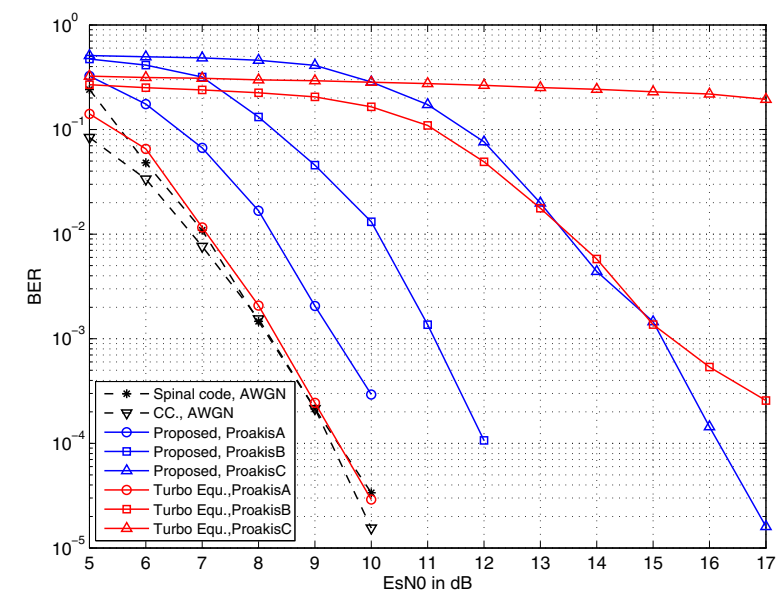

Fig. 10. The performance of proposed scheme and Turbo equalization for 16-QAM modulation
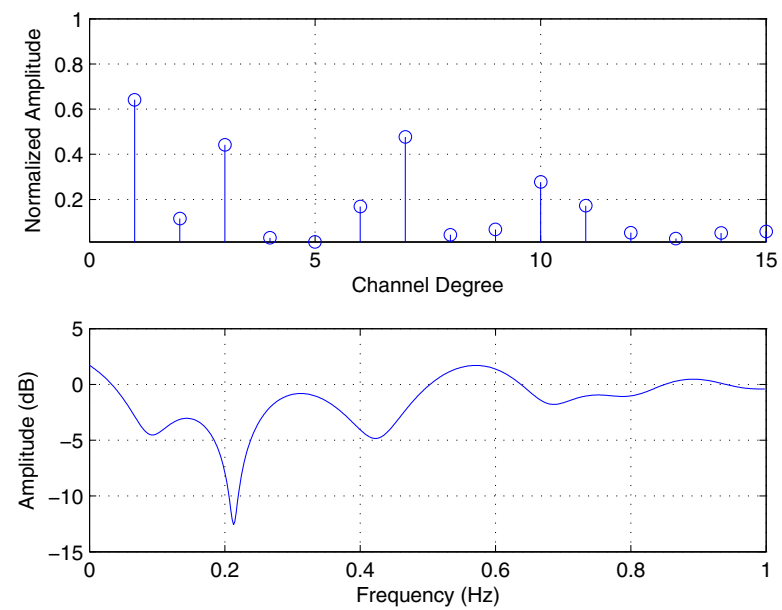

Fig. 11. The amplitude-time and amplitude-frequency responses of a shallowsea UAC

carried out in South China Sea in 2013 by IOA, CAS. The water depth of the experiment area is $5000 \mathrm{~m}$. The distance between the transmitter and the receiver is $69 \mathrm{~km}$ which is exactly in the second shadow area of the transmission loss curve. The channel is affected by a severe multipath with a delay of 100 symbol periods.

The performance comparison is illustrated in Fig.14.

\section{CONCLUSION}

This paper presents the design and performance analysis of a modified spinal code used for underwater acoustic transmissions, together with a joint equalization and decoding scheme. A tree-based suboptimal decoding algorithm is implemented with lower complexity, where decoding and equalization are performed jointly on a non-time varying underwater acoustic channel (UAC). This non-iterative receiver scheme shows stable and acceptable performance for a larger range of channels

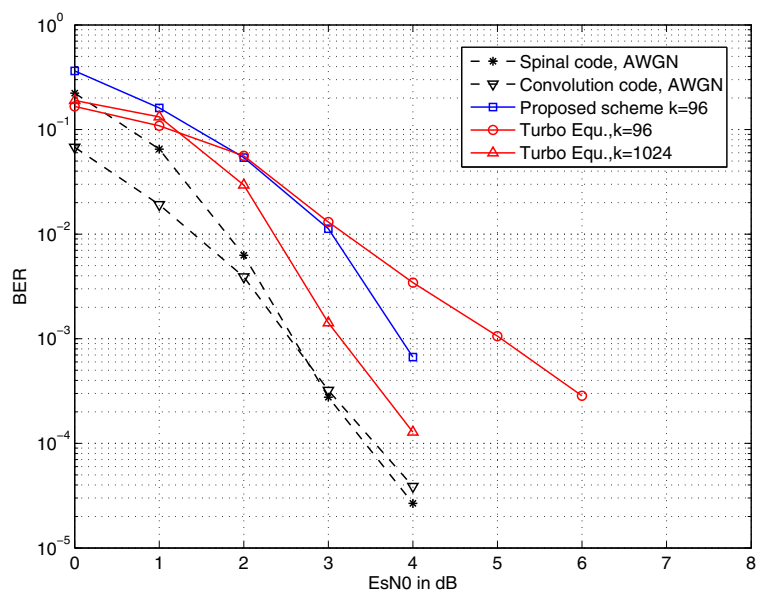

Fig. 12. The performance of the proposed scheme and Turbo equalization over shallow-sea UAC
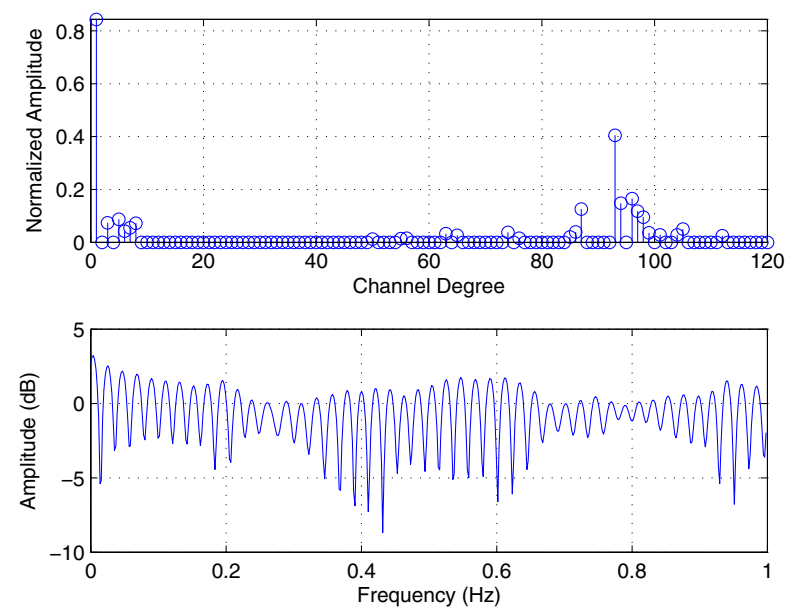

Fig. 13. The amplitude-time and amplitude-time responses of deep-sea underwater acoustic channel

when small block lengths are considered. The performance gap between the proposed scheme and the LE-MMSE turbo equalization is as large as $6 \mathrm{~dB}$ over the highly frequencyselective Proakis B channel using 16QAM modulation method.

The aim of implementing small length transmission blocks is then to provide efficient transmission schemes on fast timevarying UAC. The perspective of this work relies mainly in implementing the proposed transmission scheme for timevarying UAC and also to apply adaptive equalization for further comparisons.

\section{REFERENCES}

[1] C. Douillard, M. Jézéquel, C. Berrou, D. Electronique, A. Picart, P. Didier, and A. Glavieux, "Iterative correction of intersymbol interference: Turboequalization," Eur. Trans. Telecommun., vol. 6, no. 5, pp. 507-511, 1995.

[2] C. Laot, A. Glavieux, and J. Labat, "Turbo equalization: adaptive equalization and channel decoding jointly optimized," IEEE Journal on Selected Areas in Communications, vol. 19, no. 9, pp. 1744-1752, 2001. 


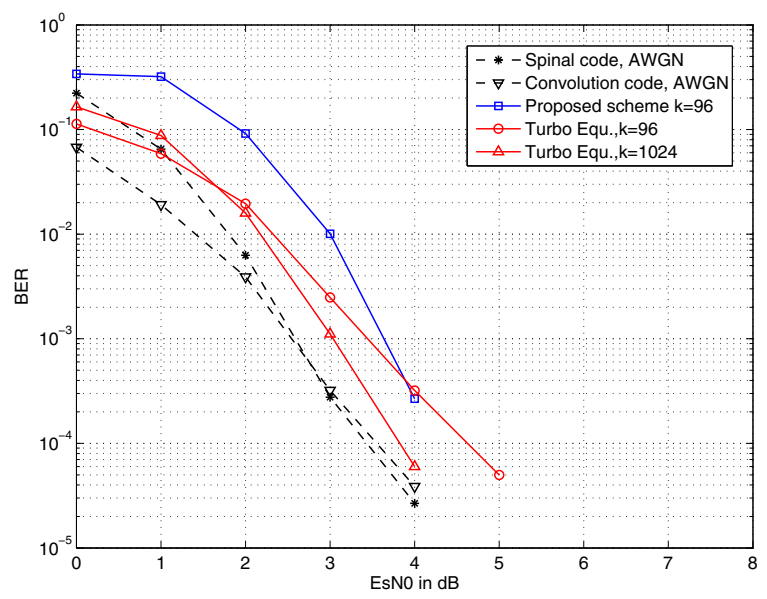

Fig. 14. The performance of proposed the scheme and Turbo equalization over deep-sea UAC

[3] M. Tuchler, R. Koetter, and A. C. Singer, "Turbo equalization: principles and new results," IEEE Transactions on Communications, vol. 50, no. 5 , pp. 754-767, May 2002.

[4] J. Perry, P. A. Iannucci, K. E. Fleming, H. Balakrishnan, and D. Shah, "Spinal codes," in Proceedings of the ACM SIGCOMM 2012 conference on Applications, technologies, architectures, and protocols for computer communication. ACM, 2012, pp. 49-60.

[5] J. Anderson and S. Mohan, "Sequential coding algorithms: A survey and cost analysis," IEEE Transactions on communications, vol. 32, no. 2, pp. 169-176, 1984

[6] L. Bahl, J. Cocke, F. Jelinek, and J. Raviv, "Optimal decoding of linear codes for minimizing symbol error rate (corresp.)," IEEE Transactions on Information Theory, vol. 20, no. 2, pp. 284-287, Mar 1974.

[7] J. G. Proakis, Digital Communications. McGraw-Hill, 1995. 